\title{
Grafting as a potent molecular tool
}

Grafting two transgenic plants triggers lateral gene transfer at the graft site but does not elicit long-distance transport of DNA into the scion or root of the graft.

Initially Ralph Bock's ambition was not to develop a new technology to make transgenic bell peppers; that interest came later. Initially the researcher from the Max Planck Institute of Molecular Plant Physiology in Potsdam, Germany was interested in the evolution of the eukaryotic cell.

"We were trying to reconstruct processes in the laboratory that normally take place only on evolutionary time scales," says Bock. "Eukaryotic cells arose through endosymbiosis. Initially these endosymbionts had large genomes," he explains, "but what is left now are just small remnants. The genes were transferred from the organellar to the nuclear genome, and this was the process we studied in detail." In previous years his team had designed selection systems that showed efficient gene transfer within a cell from chloroplasts to the nucleus. These results led Bock to ask whether this exchange is restricted to intracellular gene transfer or if genes could also travel between cells.

Together with his technician Sandra Stegemann, he designed two transgenic tobacco plants, one carrying a resistance marker and YFP in the nuclear genome, the other carrying a different resistance gene and GFP in the chloroplast genome. To facilitate genetic exchange between cells from the two different plants, they grafted one plant onto the other and then monitored the flow of genetic information by fluorescence microscopy.

The results—cells harboring both nuclear and chloroplast markers-confirmed that exchange of large pieces of DNA, possibly entire chloroplasts, takes place but only at the immediate graft site. Bock saw no evidence for long-distance transport of genes.

The mechanism responsible for the intercellular exchange of DNA is not well understood. Contrary to small nucleic acids, such as microRNAs that are known to spread systemically through the phloem from a single entry point, Bock's observations of cell-to-cell transport pointed to the involvement of plasmodesmata. These intercellular plasma channels normally allow only the passage of small molecules, such as sugars, but they can be widened by specific proteins. Bock is currently investigating whether it is indeed the plasmodesmata that are responsible for the lateral gene transport he saw in the grafts.

With the data for only short-range gene flux in grafts, Bock also provides molecular evidence that the concept of graft hybridization is untenable. "The original idea [of graft hybridization] was that you can change the heritable properties of the plant by grafting, that there is no principal difference between sexual and graft hybridization," explains Bock. This hypothesis gained notoriety in the former Soviet Union under Stalin, where the Soviet scientist Trofim Denisovich Lysenko supported it. Dissenters to the theory, also referred to as Lysenkoism, often found themselves in prison, if not worse.

Today Lysenko's tenet is largely discredited, yet there are still a few reports published that claim to see heritable changes after a plant has gone through the grafting procedure. According to Bock, these reports mainly come from breeders, and so far there is no molecular evidence for graft hybridization.

Whereas Bock's original interest lay in wanting to understand horizontal gene transfer during evolution, the results made him wonder whether grafting could also be used for moving genes into chloroplasts. Chloroplast transformation is a notoriously difficult procedure that only few plants allow. To circumvent this problem Bock envisages lateral gene transfer from the chloroplast of a species that can be easily transformed, such as tobacco, to one that is recalcitrant to transformation, such as bell pepper. After grafting, the transformed chloroplast genome would simply move into the cells of the other species.

Bock's work serves an unusually wide range of purposes: it addresses questions of evolution, provides a biotechnological application and helps to come to terms with the legacy of Lysenkoism.

\section{Nicole Rusk}

\section{RESEARCH PAPERS}

Stegemann, S. \& Bock, R. Exchange of genetic material between cells in plant tissue grafts. Science 324, 649-651 (2009). 